\title{
Evaluación del Talk Test como método para estimar la intensidad del ejercicio en niños sanos Evaluation of the Talk Test as a method to estimate exercise intensity in healthy children *Danissa Hernández, *Natalia Pacheco, *Issis Poblete, **Héctor Torres, ***Iván Rodríguez-Núñez, *Universidad Santo Tomás (Chile), ${ }^{* *}$ Universidad San Sebastián (Chile.), ${ }^{* * * U n i v e r s i d a d ~ d e ~ C o n c e p c i o ́ n ~(C h i l e) ~}$
}

Resumen. Introducción: La frecuencia cardíaca (FC) ha sido tradicionalmente utilizada para regular la intensidad del ejercicio, sin embargo, presenta limitaciones para su cuantificación sin un monitor cardiaco. En consecuencia, métodos indirectos, como el Talk Test (TT), han sido validados en adultos, no así en población infantil. El objetivo de este estudio fue determinar la relación entre el TT y los métodos objetivos para cuantificar la intensidad del ejercicio en niños sanos chilenos. Métodos: Se consideró a niños sanos de ambos géneros entre 8 y 12 años. Los sujetos realizaron dos pruebas de ejercicio incremental en escalón separadas por 7 días. El TT se incorporó en una de las dos pruebas aleatoriamente. Se midió la FC, percepción del esfuerzo (PE) y carga de trabajo, cada un minuto durante la prueba. Se comparó la FC, PE y carga de trabajo entre la respuesta positiva (TT+/+), equívoca (TT+/-) y negativa (TT-/-) del TT. Adicionalmente, se determinó la confiabilidad de los parámetros cardiorrespiratorios durante el TT. Un valor de p<.05 se consideró significativo. Resultados: Ingresaron 48 sujetos, edad $9.7 \pm 1.1$ años. Existió diferencia significativa en los parámetros cardiorrespiratorios entre las respuestas del TT (p<.0001). El \% de la FC máxima en TT+/+ fue 72.2 \pm 8.2, en TT+/- fue 87.9 \pm 8.1 y en TT-/- fue 92.0 $\pm 7.5 \%$. La confiabilidad de los parámetros cardiorrespiratorios durante el TT fue regular a excelente en ambos géneros. Conclusiones: El TT se relacionó con los parámetros cardiorrespiratorios para regular la intensidad del ejercicio en niños sanos.

Palabras claves: Ejercicio; Prueba de esfuerzo; Umbral anaeróbico; Talk test; Percepción del esfuerzo; Niños.

Abstract. Introduction: Heart rate (HR) has traditionally been used to regulate the intensity of exercise; however, its measurement is limited in the absence of a heart rate device. Consequently, indirect methods, such as the Talk Test (TT), have been validated in adults, yet not in children. The objective of this study was to determine the relationship between TT and objective methods to quantify the intensity of exercise in healthy Chilean children. Methods: Healthy children of both genders aged between 8 and 12 years old were considered for the study. The participants performed two incremental step exercise tests separated by 7 days. The TT was incorporated in one of the two tests randomly. The HR, effort perception (EP) and workload were measured every one minute during the test. HR, EP and workload were compared by positive $(\mathrm{TT}+/+)$, equivocal $(\mathrm{TT}+/-)$, and negative (TT - / -) response of the TT. Additionally, the reliability of cardiorespiratory parameters during TT was determined. A value of $\mathrm{p}<0.05$ was considered significant. Results: 48 participants aged $9.7 \pm 1.1$ years old were selected. There was a significant difference in cardiorespiratory parameters between the TT responses ( $<$ <.001). The percent of maximum HR in TT $+/+$ was $72.2 \pm 8.2 \%$, in TT $+/$ - was $87.9 \pm 8.1 \%$ and in TT $-/$ - was $92.0 \pm$ $7.5 \%$. The reliability of cardiorespiratory parameters during TT was fair to excellent in both genders. Conclusions: TT was associated to cardiorespiratory parameters to regulate exercise intensity in healthy children.

Keywords: Exercise; Exercise test; Anaerobic threshold; Talk test; Perceived exertion; Children.

\section{Introducción}

Uno de los factores que determina la eficacia de un programa de entrenamiento físico es la regulación de su intensidad. Así, diversas guías de práctica clínica y consensos de expertos sugieren la realización de ejercicio físico $(\mathrm{EF})$ de intensidad moderada a vigorosa, tres a cinco veces por semana, para mejorar la aptitud cardiorrespiratoria de la población infantil (Martinez-López, Moreno-Cerceda, Suarez-Manzano, \& Ruiz-Ariza, 2017; US. Departament of Health and Human Services, 2018; Torres-Castro et al., 2016; Yi et al., 2019).

La frecuencia cardíaca (FC) ha sido validada y ampliamente utilizada para evaluar y prescribir la intensidad del EF (Pino Ortega et al., 2019). Este parámetro presenta una estrecha relación con el $\mathrm{VO}_{2}$, principalmente cuando éste se desarrolla a intensidades moderadas (Hills, Byrne, \& Ramage, 1998). No obstante, en ausencia de un monitor cardiaco para su determinación, la FC presenta ciertas limitaciones que afectan su validez e incrementan el riesgo de error a la hora de estimar la intensidad del EF (Dunbar, Goris, Michielli, \& Kalinski, 1994). Por este motivo, diversos métodos subjetivos han sido propuestos para contrarrestar estas limitacio-

Fecha recepción: 11-03-19. Fecha de aceptación: 25-09-19 Iván Rodríguez-Núnez

ivanrodrigueznu@gmail.com nes, siendo la percepción del esfuerzo (PE) y el Talk Test (TT) los más estudiados (Álvarez Medina, Murillo Lorente, Usan Supervía, Ros Mar, \& Manonelles Marqueta, 2016; Bautista, Chirosa, Chirosa, Martin, \& Rivilla, 2016; De Lucca, Freccia, Silva, \& Oliveira, 2012; Hernández-Álvarez, del-Campo-Vecino, Martínez-de-Haro, \& Moya-Morales, 2010).

El TT consiste en evaluar la capacidad de un individuo de verbalizar un párrafo predefinido en cada nivel de intensidad durante un test de ejercicio incremental estandarizado. Tres posibles resultados pueden desencadenarse al aplicar el TT: [i] que el individuo pueda hablar confortablemente (resultado positivo o $\mathrm{TT}+/+$ ); [ii] que el individuo no esté seguro respecto a su confortabilidad (resultado equívoco o TT+/-); [iii] que el individuo no pueda verbalizar en voz alta el párrafo completo indicado (resultado negativo o TT-/-) (Reed \& Pipe, 2014).

El uso del TT se sustenta en que la ejecución de EF a una intensidad equivalente o superior al umbral ventilatorio (VT; del inglés ventilatory threshold) no permite la verbalización de frases por considerarse un punto en que la ventilación se incrementa de manera desproporcionada respecto a la carga de trabajo (Reed \& Pipe, 2014). En consecuencia, la respuesta positiva del TT $(\mathrm{TT}+/+)$ se produciría a una intensidad de ejercicio inmediatamente inferior al VT, y las otras respuestas (TT+/- y TT-/-) a intensidades de ejercicio superiores, pudiendo alcanzar incluso el umbral de compensación respiratoria (Rodriguez-Marroyo, Villa, Garcia-Lopez, \& Foster, 
2013; Woltmann et al., 2015). Si bien, el TT ha mostrado ser un método valido para regular la intensidad del EF en adultos sanos y con enfermedades cardiovasculares, este método de prescripción no ha sido estudiado en población infantil (Norman, Hopkins, \& Crapo, 2008; Rodriguez-Marroyo et al., 2013; Zanettini et al., 2013).

La aplicación efectiva del TT requiere de capacidades cognitivas que permitan al individuo memorizar las frases predefinidas e identificar el grado de esfuerzo respiratorio durante su verbalización (Reed \& Pipe, 2014). Considerando que los niños menores de 12 años presentan dificultades para traducir de forma efectiva la dimensión perceptiva del estrés fisiológico durante el ejercicio, resulta relevante estudiar la aplicabilidad del TT en este grupo etario (RodríguezNúñez \& Gatica, 2016).

Por lo precedentemente planteado, el objetivo primario de este estudio fue determinar la relación entre el TT y los métodos objetivos para cuantificar la intensidad del ejercicio en una muestra de niños sanos de Concepción, Chile. Adicionalmente, se evaluó la confiabilidad intra observador de los parámetros cardiorrespiratorios, en el nivel de intensidad de ejercicio equivalente a las diferentes respuestas del TT.

Como hipótesis primaria fue planteado que los tres estadios de este método de prescripción (TT+/+, TT+/- y TT-/-) determinan distintas intensidades de $\mathrm{EF}$ en niños sanos entre ocho y 12 años. Adicionalmente, como hipótesis secundaria fue planteado que los parámetros cardiorrespiratorios alcanzados en cada estadio del TT son reproducibles en una segunda prueba de ejercicio.

\section{Material y métodos}

\section{Diseño}

Se empleó un diseño cuasiexperimental cruzado y aleatorizado. En este diseño los individuos realizaron dos pruebas de ejercicio idénticas, en días diferentes, con una semana de intervalo. En una de las dos pruebas se incluyó aleatoriamente el TT como método de evaluación de la intensidad de ejercicio. Así, se efectuó una prueba de ejercicio con la aplicación del TT (prueba c/TT) y otra sin la aplicación del TT (prueba s/TT).

\section{Muestra de estudio}

Se consideró a niños saludables, de ambos sexos, pertenecientes a dos colegios públicos de la Ciudad de Concepción, con edades entre ocho y 12 años. Como criterio de exclusión se consideró la existencia de alguna enfermedad neurocognitiva, neuromuscular, cardiovascular o respiratoria crónica. También fueron excluidos niños con alguna enfermedad metabólica u obesidad, según criterio OMS (OMS, 2019); así como también, aquellos que practicaban alguna actividad deportiva de alto rendimiento al momento de la ejecución del estudio. La existencia de criterios de exclusión fue verificada mediante una lista de chequeo completada directamente por los padres, según protocolos previamente realizados por nuestro grupo (Rodriguez-Nunez, Navarro, Gatica, \& Manterola, 2016).

El adulto responsable (madre, padre o tutor legal) de cada uno de los participantes firmó un consentimiento informado antes de ser enrolado en el estudio. El protocolo fue aprobado por el comité ético científico de la Universidad Santo Tomás de Concepción, Chile.

\section{Protocolo experimental}

El protocolo se llevó a cabo en tres sesiones separadas por una semana de intervalo entre ellas. El estudio se realizó entre las nueve y 11 horas, en las instalaciones deportivas de los colegios.

En la primera sesión se midieron las variables antropométricas y los parámetros cardiorrespiratorios de reposo. El peso y la estatura se midió con una balanza análoga (JASM-2003B) y cinta métrica (Stabila Clase II) respectivamente. El índice de masa corporal se calculó de acuerdo al índice de Quetelet (peso/estatura ${ }^{2}$ ). Por su parte, la presión arterial se midió en la muñeca de manera no invasiva y con un monitor de presión digital (Braun Vital Scan 1 BBP 2000) y la FC con un monitor cardíaco Polar (rs800cx). La FC máxima teórica se calculó según la fórmula de Tanaka: 208 - (0,7 x edad) (Machado \& Denadai, 2011). Finalmente, la PE se midió con la escala EPInfant, según las recomendaciones publicadas por Rodríguez-Núñez et al. (Rodríguez et al., 2015).

Adicionalmente, durante esta sesión los participantes del estudio fueron familiarizados con el test de ejercicio y fueron instruidos respecto a la aplicación del TT según el protocolo publicado por Reed et al. (Reed \& Pipe, 2014).

En cada sesión siguiente se realizó un test de ejercicio incremental, incorporando aleatoriamente en una de las dos instancias la aplicación del TT. Como prueba de ejercicio se utilizó el test de escalón incrementa de Chester (TChester), el cual, ha mostrado adecuadas propiedades clinimétricas en niños chilenos (Rodríguez-Núñez, Gatica Solís, \& Navarro Tapia, 2016).

El TChester consiste en subir y bajar un escalón de $20 \mathrm{~cm}$ de altura a un ritmo y velocidad proporcionado por una señal sonora (metrónomo). La intensidad del ejercicio se estandariza en ciclos, que equivale a un ascenso y un descenso con ambos pies (cuatro pasos). Originalmente, la prueba de ejercicio posee cinco niveles intensidad con una duración de dos minutos cada uno, iniciando con una intensidad de 15 ciclos/minuto (60 pasos) e incrementos de cinco ciclos/minuto por cada nivel (20 pasos), hasta alcanzar 35 ciclos $/ \mathrm{mi}$ nuto (140 pasos). No obstante, para nuestro estudio se adicionaron tres niveles de intensidad con el propósito de asegurar que cada participante alcanzara su FC peak. En consecuencia, la prueba contó con ocho niveles, alcanzando una intensidad máxima de 50 ciclos/minuto (200 pasos).

Se registró la PE y la FC antes y después del TChester. Durante la prueba, ambas variables se registraron por dos evaluadores independientes, en los últimos 20 segundos de cada minuto.

\section{Talk Test}

Para la realización del TT se instruyó a los participantes a recitar en voz alta un párrafo estándar durante los últimos 30 segundos de cada nivel de intensidad del TChester. Se consideró como párrafo estándar a las primeras 31 palabras del «Himno nacional de Chile». Posteriormente, luego de recitar este párrafo se formuló la pregunta ¿Puedes hablar cómodamente? Considerado tres posibles respuestas: [i] res- 
puesta positiva (TT+/+), cuando el individuo recitó el párrafo completo sin dificultad; [ii] respuesta equívoca (TT +/-), cuando el individuo recitó el párrafo completo con dificultad; y [iii] respuesta negativa (TT -/-), cuando el individuo no fue capaz de recitar el párrafo completo (Reed \& Pipe, 2014). Se registró la FC, la PE y el nivel del TChester, equivalente a cada respuesta del TT. Como TT+/+ se consideró los datos del último nivel del TChester en que el individuo pudo recitar el párrafo establecido sin dificultad y como TT+/- se consideró al primer nivel del TChester en que el individuo manifestó percibir dificultad para completar la prueba.

\section{Análisis estadístico}

El tamaño muestral se estimó a partir de resultados de estudios previos realizados en adultos, en los cuales se observa una diferencia aproximada de 13 lat/min entre el TT-/+ y el TT-/- y una desviación estándar de 10 lat/min (RodriguezMarroyo et al., 2013). Así, considerando un riesgo alfa de 5\% y beta de $5 \%$, el número calculado fue de 10 sujetos por género ( $n=20)$. Sin embargo, considerando la inexistencia de datos en población infantil que permitan estimaciones más precisas del tamaño muestral, y para aminorar a probabilidad de error tipo II, se decidió arbitrariamente trabajar con un tamaño muestral mínimo equivalente al doble de la muestra calculada $(\mathrm{n}=40)$.

Se realizó análisis exploratorio de los datos con evaluación de normalidad, mediante el test de Shapiro Wilk. Una vez verificada la normalidad de los datos cuantitativos se realizó estadística descriptiva con cálculo de promedio y desviación estándar, así como mediana y rango intercuartílico para el numero de niveles desarrollados durante el TChester.

Para comparar las diferencias entre géneros se utilizó la prueba t-Student para muestras independientes en las variables cuantitativas y la prueba U-Mann Whitney en las variables cualitativas. Para evaluar las diferencias de los resultados descriptivos entre el TChester s/TT y c/TT se empleó la prueba t-Student para muestras pareadas en las variables cuantitativas y la prueba de Wilcoxon en las variables cualitativas. Adicionalmente, se empleó ANOVA de medidas repetidas para comparar la FC y la PE entre los niveles del TChester, así como entre los niveles de respuesta del TT.

Finalmente, la confiabilidad de la FC y la PE equivalente a cada respuesta del TT se evaluó mediante el cálculo del coeficiente de correlación intraclase (CCI). Sus resultados se expresaron en valor absoluto y 95\% de intervalo de confianza (95\%IC). Un CCI mayor a .75 fue considerado como excelente, entre .74 y .41 regular a bueno; y un CC inferior a .40 fue considerado como pobre (Fleiss, Levin, \& Paik, 2003).

Todos los análisis se efectuaron en el software estadístico MedCalc versión 18.11.3 (MedCalc Software bvba, Ostend, Belgium; 2019), considerando un valor de $p<.05$ como estadísticamente significativo.

\section{Resultados}

$\mathrm{Al}$ estudio ingresaron 48 sujetos (52.1\% hombres), con un promedio de edad de $9.7 \pm 1.1$ años. No existió diferencia significativa en cuanto a las características antropométricas y variables cardiovasculares de reposo entre géneros (Tabla 1).
Todos los sujetos completaron ambas pruebas de ejercicio. No existieron diferencias estadísticas en el rendimiento del TChester entre la prueba s/TT y c/TT (Tabla 2).

En relación al análisis por género, las mujeres realizaron menos niveles del TChester que los hombres, en ambas pruebas (prueba s/TT, $p=.0374$; prueba c/TT, $p=.0104$ ). No existió diferencia significativa en el resto de las variables cardiorrespiratorias máximas al final del TChester entre hombres y mujeres (Tabla 2).

En la figura 1 se muestra el incremento de la FC y la PE durante la prueba s/TT y c/TT. En ella se observa una mayor FC y PE en las mujeres comparado a los hombres, principalmente en los estadios intermedios del TChester.

En relación al análisis de los parámetros de intensidad del ejercicio durante el TT se observaron diferencias estadísticamente significativas en todas las variables analizadas (Figura 2 a-d). Así, en la muestra total la FC en TT+/+ fue 145.3 \pm 16.6 lat/min (\% FC máx: 72.2 $\pm 8.2 \%$ ), en TT+/- de 176.8 \pm 16.4lat/min (\%FCmáx: 87.9 $\pm 8.1 \%$ ) y en TT-/-de 185.2 \pm 15.2 lat/min (FCmáx: $92.0 \pm 7.5 \%)(p<.0001)$. Por su parte, la $\mathrm{PE}$ en TT+/+ fue de $3.9 \pm 1.9$, en TT+/- de $6.9 \pm 2.1$ y en TT-/ - de $8.4 \pm 1.8(p<.0001)$.

Respecto a la carga de trabajo expresada en número de pasos por minuto (pasos/min), la muestra total en $\mathrm{TT}+/+$ alcanzó $109.6 \pm 22.6$ pasos $/ \mathrm{min}$, en TT $+/-158.7 \pm 21.7$ pasos/ min y en TT-/- $180.0 \pm 24.0$ pasos/min $(p<.0001)$. Solo se observó diferencia significativa entre géneros en TT+/-(hombres: $166.4 \pm 19.9$ pasos/min y mujeres: $151.6 \pm 21.2$ pasos/ min; $p=.0194$ ) y en TT-/-(hombres: $190.5 \pm 20.4$ pasos/min y mujeres: $170.5 \pm 23.3$ pasos $/ \mathrm{min} ; p=.0062$ ) (Figura 2d).

La confiabilidad de la FC y la PE en cada una de las respuestas del TT se muestra en la tabla 3. En ambas variables la confiabilidad fue regular a excelente en la muestra

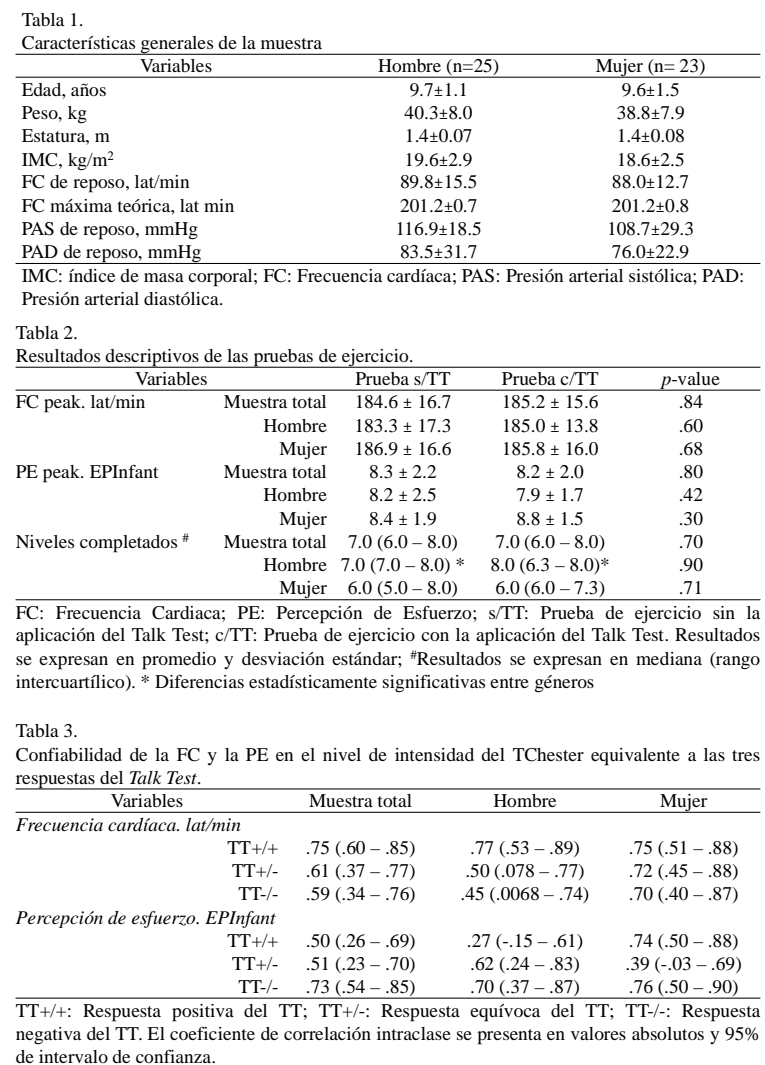


total. Solo la PE mostró una confiabilidad débil y no estadísticamente significativa en el TT+/+ en hombres y en el TT+/- en las mujeres.
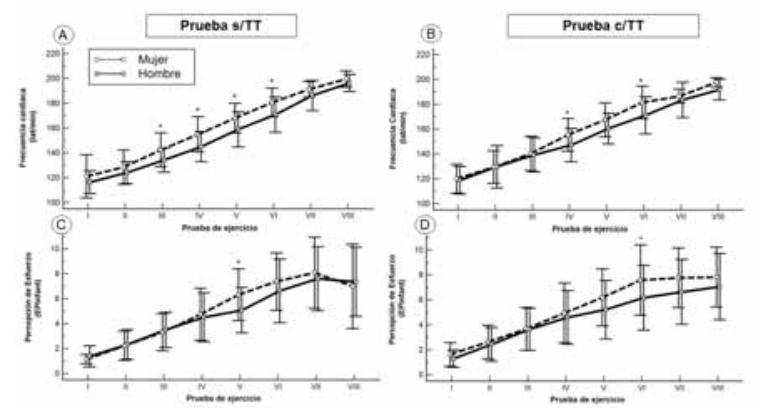

Figura
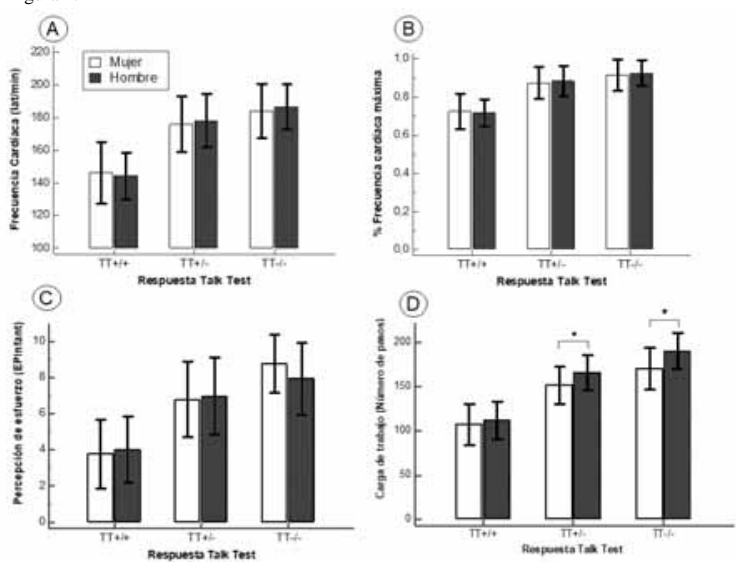

Figura 2.

\section{Discusión}

Los principales hallazgos de este estudio apoyan la hipótesis de que el TT podría ser un método valido para la estimación de distintas intensidades de ejercicio en niños sanos, de edades entre ocho y 12 años. Así, nuestros resultados concuerdan con estudios previos, en los que se ha constatado que las tres respuestas del TT podrían identificar niveles de intensidad baja, moderada y alta durante una prueba de ejercicio incremental (De Lucca et al., 2012; Gillespie, McCormick, Mermier, \& Gibson, 2015; Persinger, Foster, Gibson, Fater, \& Porcari, 2004; Reed \& Pipe, 2014; Woltmann et al., 2015).

En nuestro estudio la FC registrada en las tres etapas del TT fue superior a la reportada en estudios realizados en adultos. Gillespie et al., registraron una intensidad equivalente al 68,6\%, 78,5\% y 83,1\% de la FC máxima en TT+/+, TT+/-yTT/- respectivamente, en adultos durante una prueba incremental en cicloergómetro (Gillespie et al., 2015). Resultados similares se observaron cuando la FC fue expresada en relación a la FC de reserva (Persinger et al., 2004). Por su parte, Rodríguez-Marroyo et al, registraron en ciclistas entrenados (20 \pm 2 años) una FC de 132, 148 y 176 lat/min en los respectivos estadios del TT (Rodriguez-Marroyo et al., 2013).

Este fenómeno podría ser explicado por la existencia demostrada de un mayor umbral anaeróbico en los niños prepuberales y puberales comparado a individuos adultos, debido una menor producción de lactato sanguíneo y muscular durante el ejercicio submáximo en este grupo etario (Armstrong \& Welsman, 1994; Chicharro \& Vaquero, 2006).
Así, estudios realizados en niños entre nueve y 10 años revelaron que el VT podría desencadenarse entre el 64\% y $71 \%$ del $\mathrm{VO}_{2}$ máx y a una FC entre 155 y 160 lat/min, equivalente al 80\% de la FC máx aproximadamente (Amedro et al., 2018; Mahon, Gay, \& Stolen, 1998; Reybrouck, Weymans, Stijns, Knops, \& van der Hauwaert, 1985).

En nuestro estudio la FC en el TT+/+ fue de 145.3 lat $/ \mathrm{min}$ (72\% de la FC máx), lo cual, se encontraría por debajo del nivel de intensidad del VT que ha sido reportada en niños (Amedro et al., 2018; Mahon et al., 1998). Esto concuerda con los estudios de validación del TT, en donde se ha demostrado que la respuesta positiva de la prueba $(\mathrm{TT}+/+)$ produce niveles de intensidad de ejercicio inferiores al VT. A diferencia de las otras respuestas (TT+/- y TT-/-), las cuales, han sido documentadas en intensidades de ejercicio más cercanas al umbral de compensación respiratoria (Gillespie et al., 2015; Reed \& Pipe, 2014; Rodriguez-Marroyo et al., 2013).

Por otra parte, respecto al esfuerzo percibido en las diferentes respuestas del TT, se registró una PE de 3.9, 6.9 y 8.4 en la respuesta positiva, equívoca y negativa respectivamente. Son escasos los estudios que han evaluado la PE durante los estadios del TT mediante escalas con un rango de descriptores numéricos entre 0 y 10 . En el estudio de Rodríguez-Marroyo et al, se observó una diferencia aproximada de un punto por debajo de lo registrado en nuestro estudio en cada nivel del TT (Rodriguez-Marroyo et al., 2013). Este fenómeno es consistente con el comportamiento de la FC durante el TT y concuerda con estudios previos realizados por nuestro grupo, donde se ha establecido la fuerte interdependencia entre la FC y la PE (medida con la escala EPInfant) durante una prueba de ejercicio incremental en escalón y cinta rodante (Rodriguez-Nunez \& Manterola, 2016; Rodríguez-Núñez, Jerez, et al., 2016).

Respecto a la confiabilidad de la FC en los niveles del TChester equivalente a las diferentes respuestas del TT, los resultados mostraron un CCI entre .59 y .75 en muestra total. Si bien es cierto, no existió diferencia estadísticamente significativa entre los distintos grupos estudiados, se constató una mayor reproducibilidad en TT+/+ comparado a TT+/-y TT-/-; diferencia que fue más pronunciada en los hombres respecto a las mujeres. Estos resultados discrepan con observaciones realizadas en adultos, en quienes se ha observado una fuerte concordancia de los parámetros de estrés fisiológico (CCI > .9), tanto en TT+/- como en TT-/-(Ballweg et al., 2013). En este contexto, es probable que factores atribuidos a las características de la muestra estudiada y a la naturaleza de la prueba de ejercicio hayan influido en la confiabilidad de la FC en los niveles del TT. Estudios previos han revelado la existencia de un alto nivel de variabilidad en la confiabilidad de la FC durante el TChester en niños sanos entre ocho y 15 años, registrándose CCI entre .70 y .90 (Rodríguez-Núñez, Gatica Solís, et al., 2016).

En relación a la confiabilidad de la PE, los más altos CCI fueron observados en TT-/-, tanto en hombres, como en mujeres (.70 y .76 respectivamente). Este resultado concuerda con algunos estudios previos que han reportado niveles similares de confiabilidad durante el TChester (RodriguezNunez \& Manterola, 2016). De forma interesante, los resultados revelaron que la confiabilidad es mayor en el nivel de 
intensidad equivalente a la respuesta negativa del TT (TT-/ -), lo cual, es consistente con estudios que sugieren una mayor confiabilidad en la medición de la PE, a medida que incrementa la intensidad del ejercicio (Barroso, Cardoso, do Carmo, \& Tricoli, 2014; Rodriguez-Nunez \& Manterola, 2016). Por el contrario, en esfuerzos de baja intensidad los estudios han revelado una menor concordancia inter observador y una menor correlación con los niveles de actividad física objetivada mediante acelerometría (Carcamo-Oyarzun, Carrasco-Alarcon, Espinoza Silva, \& Martinez-Salazar, 2019; Rodriguez-Nunez \& Manterola, 2016).

Respecto a las aplicaciones prácticas de estos resultados, es posible sostener que el mayor nivel de intensidad de ejercicio en el que se consiga verbalizar sin dificultad un discurso preestablecido, en nuestro caso, el «Himno Nacional de Chile»; podría ser desencadenado en niveles inmediatamente inferiores al VT en niños. Este punto metabólico ha sido reconocido como zona óptima de entrenamiento aeróbico en diversas poblaciones y diversos contextos (Services, 2018; Torres-Castro et al., 2016). Por este motivo el TT podría ser un método aplicable y efectivo para dosificar la intensidad de ejercicio en niños sanos, tal como ha sido demostrado en adultos (De Lucca et al., 2012; Gillespie et al., 2015; Persinger et al., 2004; Reed \& Pipe, 2014; Woltmann et al., 2015; Zanettini et al., 2013).

Respecto a las limitaciones del presente estudio, vale la pena mencionar que la reproducción de la intensidad del ejercicio mediante mecanismos perceptuales depende estrechamente del desarrollo cognitivo, incluso se ha demostrado contundentemente que recién a partir de los ocho años los niños podrían discriminar cuatro o más niveles de intensidad de ejercicio en un ensayo de reproducción perceptual (Groslambert \& Mahon, 2006); por este motivo, los alcances de los resultados de nuestro estudio no podrían extenderse a edades inferiores a ocho años.

Por otra parte, la no determinación directa del $\mathrm{VO}_{2}$ solo permitió establecer la relación entre el VT y el TT desde un plano especulativo a partir de la evidencia publicada previamente, lo que no permite descartar que la relación entre ambas variables sea de una naturaleza diferente a la sugerida en este estudio.

Finalmente, es posible concluir que el TT se asoció a los parámetros objetivos de regulación de la intensidad del ejercicio en la muestra de niños estudiada. Así, mientras que la respuesta positiva del TT $(\mathrm{TT}+/+)$ se desencadenó en una zona de la FC relacionada con ejercicio aeróbico, la respuesta equívoca (TT+/-) y negativa del TT (TT-/-) se desencadenaron en zonas de FC relacionadas con el VT o superiores al umbral anaeróbico.

Por otra parte, la confiabilidad de FC y PE en las distintas respuestas del TT fue regular a excelente, lo cual, dependió del parámetro cardiorrespiratorio analizado, el tipo de respuesta del TT y el género.

Futuros estudios deben ser realizados para confirmar el potencial del TT para regular y dosificar la intensidad del ejercicio en niños sanos entre ocho y 12 años.

\section{Referencias}

Álvarez Medina, J., Murillo Lorente, V., Usan Supervía, P.,
Ros Mar, R., \& Manonelles Marqueta, P. (2016). Percepción subjetiva como método de control de la intensidad en fútbol sala Retos(30), 9-14. Retrieved from https:// recyt.fecyt.es/index.php/retos/article/view/35128

Amedro, P., Gavotto, A., Guillaumont, S., Bertet, H., Vincenti, M., De La Villeon, G., . . . Matecki, S. (2018). Cardiopulmonary fitness in children with congenital heart diseases versus healthy children. Heart, 104(12), 10261036. doi:10.1136/heartjnl-2017-312339

Armstrong, N., \& Welsman, J. R. (1994). Assessment and interpretation of aerobic fitness in children and adolescents. Exerc Sport Sci Rev, 22, 435-476.

Ballweg, J., Foster, C., Porcari, J., Haible, S., Aminaka, N., \& Mikat, R. P. (2013). Reliability of the talk test as a surrogate of ventilatory and respiratory compensation thresholds. J Sports Sci Med, 12(3), 610-611.

Barroso, R., Cardoso, R. K., do Carmo, E. C., \& Tricoli, V. (2014). Perceived exertion in coaches and young swimmers with different training experience. Int J Sports Physiol Perform, 9(2), 212-216. doi:10.1123/ijspp.20120356

Bautista, I. J., Chirosa, I. J., Chirosa, L. J., Martin, I., \& Rivilla, J. (2016). RPE y velocidad como marcadores de intensidad en el press de banca. Rev Int Med Cienc Act Fis Deporte(62). doi:10.15366/rimcafd2016.62.004

Carcamo-Oyarzun, J., Carrasco-Alarcon, V., Espinoza Silva, J. M., \& Martinez-Salazar, C. (2019). La intensidad del esfuerzo en clases de Educación Física. Relación entre percepción y cuantificación objetiva de estudiantes y profesores. Retos(36), 384-390. Retrieved from https:// recyt.fecyt.es/index.php/retos/article/view/67925/43829

Chicharro, J. L., \& Vaquero, A. F. (2006). Transición aeróbicaanaeróbica: concepto, bases fisiológicas y aplicaciones. In M. Panamericana (Ed.), Fisiología del Ejercicio (3a ed., pp. 987). Buenos Aires; Madrid.

De Lucca, L., Freccia, G. W., Silva, A. E. L. e., \& Oliveira, F. R. d. (2012). Talk test como método para controle da intensidade de exercício. Rev Bras Cineantropom Desempenho Hum, 14, 114-124. Retrieved from http:// www.scielo.br/scielo.php?script=sci_arttext\&pid=S198000372012000100012\&nrm=iso

Dunbar, C. C., Goris, C., Michielli, D. W., \& Kalinski, M. I. (1994). Accuracy and reproducibility of an exercise prescription based on Ratings of Perceived Exertion for treadmill and cycle ergometer exercise. Percept Mot Skills, 78(3 Pt 2), 1335-1344. doi:10.2466/ pms.1994.78.3c.1335

Fleiss, J. L., Levin, B., \& Paik, M. C. (2003). Measurement of Interrater Agreement. In I. Jhon Wiley \& Sons (Ed.), Statistical Methods for Rates and Proportions (3th ed., pp. 604).

Gillespie, B. D., McCormick, J. J., Mermier, C. M., \& Gibson, A. L. (2015). Talk test as a practical method to estimate exercise intensity in highly trained competitive male cyclists. J Strength Cond Res, 29(4), 894-898. doi:10.1519/ jsc.0000000000000711

Groslambert, A., \& Mahon, A. D. (2006). Perceived exertion : influence of age and cognitive development. Sports Med, 36(11), 911-928. doi:10.2165/00007256-200636110-00001

Hernández-Álvarez, J. L., del-Campo-Vecino, J., Martínez-de- 
Haro, V., \& Moya-Morales, J. M. (2010). Percepción de esfuerzo en Educación Física y su relación con las directrices sobre actividad física. Rev Int Med Cienc Act Fis Deporte, 10(40), 609-619. Retrieved from https:// www.redalyc.org/articulo.oa?id=54222122007

Hills, A. P., Byrne, N. M., \& Ramage, A. J. (1998). Submaximal markers of exercise intensity. J Sports Sci, 16 Suppl, S7176. doi:10.1080/026404198366696

Machado, F. A., \& Denadai, B. S. (2011). Validade das equações preditivas da frequência cardíaca máxima para crianças e adolescentes. Arq Bras Cardiol, 97, 136-140. Retrieved from http://www.scielo.br/ scielo.php?script=sci_arttext \&pid=S0066782X2011001100007\&nrm=iso

Mahon, A. D., Gay, J.A., \& Stolen, K. Q. (1998). Differentiated ratings of perceived exertion at ventilatory threshold in children and adults. Eur J Appl Physiol Occup Physiol, 78(2), 115-120. doi:10.1007/s004210050395

Martinez-López, E. J., Moreno-Cerceda, J., Suarez-Manzano, S., \& Ruiz-Ariza, A. (2017). Efecto y satisfacción de un programa de actividad física controlada por pulsómetro en el índice de masa corporal de escolares con sobrepesoobesidad Retos, 0(33), 179-184. Retrieved from https:// recyt.fecyt.es/index.php/retos/article/view/58019/36511

Norman, J. F., Hopkins, E., \& Crapo, E. (2008). Validity of the counting talk test in comparison with standard methods of estimating exercise intensity in young healthy adults. J Cardiopulm Rehabil Prev, 28(3), 199-202. doi:10.1097/ 01.HCR.0000320072.89093.0d

Organización Mundial de la Salud. (2019). ¿Qué son el sobrepeso y obesidad? Estrategia mundial sobre régimen alimentario, actividad física y salud. Retrieved from https://www.who.int/dietphysicalactivity/ childhood_what/es/

Persinger, R., Foster, C., Gibson, M., Fater, D. C., \& Porcari, J. P. (2004). Consistency of the talk test for exercise prescription. Med Sci Sports Exerc, 36(9), 1632-1636.

Pino Ortega, J., Bastida Castillo, A., Oliva Lozano, J. M., Rojas Valverde, D., Reche Soto, P., \& Gómez Carmona, C. D. (2019). Comparación de dos métodos de detección para el registro de la frecuencia cardíaca: banda torácica vs camiseta técnica. Retos, 36(36), 469-473. Retrieved from https://recyt.fecyt.es/index.php/retos/article/view/68676/ 43949

Reed, J. L., \& Pipe, A. L. (2014). The talk test: a useful tool for prescribing and monitoring exercise intensity. Curr Opin Cardiol, 29(5), 475-480. doi:10.1097/ hco.0000000000000097

Reybrouck, T., Weymans, M., Stijns, H., Knops, J., \& van der Hauwaert, L. (1985). Ventilatory anaerobic threshold in healthy children. Age and sex differences. Eur J Appl Physiol Occup Physiol, 54(3), 278-284.

Rodriguez-Marroyo, J.A., Villa, J. G, Garcia-Lopez, J., \& Foster, C. (2013). Relationship between the talk test and ventilatory thresholds in well-trained cyclists. J Strength Cond Res, 27(7), 1942-1949. doi:10.1519/ JSC.0b013e3182736af3

Rodriguez-Nunez, I., \& Manterola, C. (2016). Concurrent validity and interobserver reliability of the EPInfant pediatric perceived exertion rating scale among healthy
Chilean children. Arch Argent Pediatr, 114(4), 343-354. doi:10.5546/aap.2016.343

Rodriguez-Nunez, I., Navarro, X., Gatica, D., \& Manterola, C. (2016). Effect of abdominal muscle training on respiratory muscle strength and forced expiratory flows in sedentary, healthy adolescents. Arch Argent Pediatr, 114(5), 434440. doi:10.5546/aap.2016.434

Rodríguez-Núñez, I., \& Gatica, D. (2016). Percepción de esfuerzo durante el ejercicio: ¿Es válida su medición en la población infantil? Rev Chil Enferm Respir, 32(1), 25-33.

Rodríguez-Núñez, I., Gatica Solís, D., \& Navarro Tapia, X. (2016). Rendimiento de las variables cardiorrespiratorias durante el test de Chester en niños sanos chilenos. Revista Cubana de Pediatría, 88(3), 310-322. Retrieved from h t t p : / / s c i e l o . s l d. c u / scielo.php?script=sci_arttext $\&$ pid $=$ S 0034 75312016000300005\&nrm=iso

Rodríguez-Núñez, I., Jerez, R., Mora, A., Mellado, D., García, M., Gatica, D., ... Manterola, C. (2016). Evaluación de la Escala EPInfant durante una prueba de ejercicio incremental en cinta rodante. Rev Chil Enferm Respir, 32(3), 155-159.

Rodríguez, I., Zenteno, D., Cisternas, L., Rodríguez, P., Reyes, G., \& Troncoso, K. (2015). Construcción y evaluación de EPInfant: una escala para la medición del esfuerzo percibido en población pediátrica. Arch Argent Pediatr, 113(6), 550-557. Retrieved from https://dx.doi.org/10.5546/ aap. 2015.550

Torres-Castro, R., Zenteno, D., Rodríguez-Núñez, I., Villarroel, G., Alvarez, C., Gatica, D., . . Caviedes, I. (2016). Guías de rehabilitación respiratoria en niños con enfermedades respiratorias crónicas: Actualización 2016. Neumol Pediátr, 11(3), 114-131.

US. Departament of Health and Human Services. (2018). Physical Activity Guidelines for Americans. Washington, DC: Departament of Health and Human Services. Retrieved from https://health.gov/paguidelines/second-

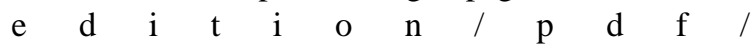
Physical_Activity_Guidelines_2nd_edition.pdf

Woltmann, M. L., Foster, C., Porcari, J. P., Camic, C. L., Dodge, C., Haible, S., \& Mikat, R. P. (2015). Evidence that the talk test can be used to regulate exercise intensity. J Strength Cond Res, 29(5), 1248-1254. doi:10.1519/ jsc.0000000000000811

Yi, D. Y., Kim, S. C., Lee, J. H., Lee, E. H., Kim, J. Y., Kim, Y. J., ... Yang, H. R. (2019). Clinical Practice Guideline for the Diagnosis and Treatment of Pediatric Obesity: Recommendations from the Committee on Pediatric Obesity of the Korean Society of Pediatric Gastroenterology Hepatology and Nutrition. Pediatr Gastroenterol Hepatol Nutr, 22(1), 1-27. doi:10.5223/ pghn.2019.22.1.1

Zanettini, R., Centeleghe, P., Franzelli, C., Mori, I., Benna, S., Penati, C., \& Sorlini, N. (2013). Validity of the Talk Test for exercise prescription after myocardial revascularization. Eur J Prev Cardiol, 20(2), 376-382. doi:10.1177/ 2047487312438982 